\title{
Observation of Biexcitons in Nanocrystal Solids in the Presence of Photocharging
}

\author{
Ahmet Fatih Cihan, ${ }^{\dagger}$ Pedro Ludwig Hernandez Martinez, ${ }^{+, \neq}$Yusuf Kelestemur, $^{\dagger}$ Evren Mutlugun, ${ }^{t, \neq}$ and \\ Hilmi Volkan Demir ${ }^{*,+, *}$ \\ ${ }^{\dagger}$ Department of Electrical and Electronics Engineering, Department of Physics, UNAM Institute of Materials Science and Nanotechnology, Bilkent University, \\ Ankara 06800 Turkey, and ${ }^{\ddagger}$ School of Electrical and Electronic Engineering, School of Physical and Mathematical Sciences, Nanyang Technological University, \\ Nanyang Avenue, Singapore 639798, Singapore
}

\begin{abstract}
In nanocrystal quantum dots (NQDs), generating multiexcitons offers an enabling tool for enhancing NQD-based devices. However, the photocharging effect makes understanding multiexciton kinetics in NQD solids fundamentally challenging, which is critically important for solid-state devices. To date, this lack of understanding and the spectral-temporal aspects of the multiexciton recombination still remain unresolved in solid NQD ensembles, which is mainly due to the confusion with recombination of carriers in charged NQDs. In this work, we

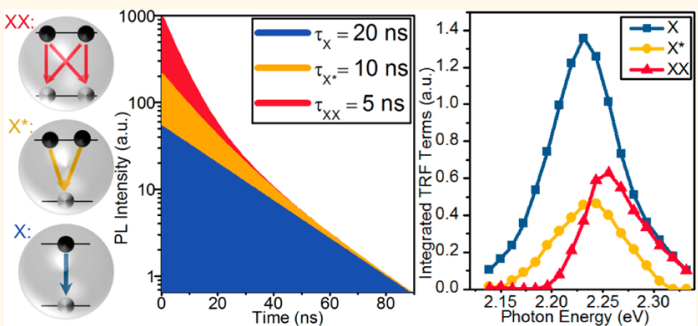
reveal the spectral-temporal behavior of biexcitons (BXs) in the presence of photocharging using near-unity quantum yield CdSe/CdS NQDs exhibiting substantial suppression of Auger recombination. Here, recombinations of biexcitons and single excitons $(X s)$ are successfully resolved in the presence of trions in the ensemble measurements of time-correlated single-photon counting at variable excitation intensities and varying emission wavelengths. The spectral behaviors of BXs and Xs are obtained for three NQD samples with different core sizes, revealing the strength tunability of the $X-X$ interaction energy in these NQDs. The extraction of spectrally resolved $X, B X$, and trion kinetics, which are otherwise spectrally unresolved, is enabled by our approach introducing integrated time-resolved fluorescence. The results are further experimentally verified by cross-checking excitation intensity and exposure time dependencies as well as the temporal evolutions of the photoluminescence spectra, all of which prove to be consistent. The BX and X energies are also confirmed by theoretical calculations. These findings fill an important gap in understanding the spectral dynamics of multiexcitons in such NQD solids under the influence of photocharging effects, paving the way to engineering of multiexciton kinetics in nanocrystal optoelectronics, including NQD-based lasing, photovoltaics, and photodetection.
\end{abstract}

KEYWORDS: semiconductor quantum dots - multiexciton generation · multiexciton recombination · biexciton · trion time-resolved fluorescence

$\mathrm{P}$ hotogeneration and recombination of multiexcitons (MEs) in semiconductor nanocrystal quantum dots (QDs) have recently attracted significant scientific interest as a possible means to improve the performances of QDs in device applications. High surface qualities and small density of defect sites with engineered band structures of recently developed core/shell QDs, especially those with core/shell materials of $\mathrm{CdSe} / \mathrm{CdS}^{1-7}$ and $\mathrm{CdTe} / \mathrm{CdSe}{ }^{8-10}$ enabled better multiexciton generation (MEG) and recombination (MER) performances, encouraging researchers in this field. In very recent reports, the MEG phenomenon has indeed been shown to be very promising, especially for improving solar energy conversion efficiencies. ${ }^{11-21}$ It has also been demonstrated that even over $100 \%$ peak external quantum efficiency is possible for QD solar cells exploiting the MEG concept. ${ }^{11}$ Another important research area that relies on MEG and MER processes is the utilization of QDs as the gain medium for lasing applications. ${ }^{7,22-24}$ Easy spectral tunability and comparatively easy synthesis of QDs via wet chemistry make them very convenient candidates for lasing applications. In addition to photovoltaic and lasing applications, the MEG concept has also been shown to significantly improve photodetector device performances. ${ }^{25}$ For all of these application areas, the most pronounced limiting effect is the nonradiative Auger recombination (AR) of MEs. Therefore,

\section{* Address correspondence to hvdemir@ntu.edu.sg volkan@bilkent.edu.tr.}

Received for review November 12, 2012 and accepted May 28, 2013.

Published online May 28, 2013 $10.1021 / \mathrm{nn} 305259 \mathrm{~g}$

() 2013 American Chemical Society 
the suppression of $A R$ is also the subject of intense research in order to benefit from the MEs most effectively. ${ }^{2,4,5,7,8,26}$ Until very recently, MER had been believed to be fully nonradiative as AR. However, it was shown that MERs do not have to be nonradiative and could even be made fully radiative for some special QDs. ${ }^{5,27}$

Although there are a great number of strong experimental and theoretical studies in the literature on MEG on a wide variety of material and shape combinations, the temporal and spectral aspects of the generation and recombination of MEs still remain under debate. Especially the confusion of the MER with the recombination of carriers in a charged QD has caused a number of different and contradicting results to be reported in the literature. ${ }^{18,28-32}$ The confusion of the recombination in charged QDs and the MER has previously been addressed by the prevention of charging of the QDs by effectively exposing each QD to pulses of excitation less frequently through stirring or flowing the samples in solution. ${ }^{20,33-35}$ Although this approach has led to very important explorations and clarifications about MEs, a deeper understanding of MEs is necessary, because avoiding the photocharging of the QDs in practical applications, such as QD lasing and solar energy conversion based on MEG, where typically thin films of QDs are used in solid form, may not be generally possible. Therefore, the behavior of the MEs in the presence of photocharging in solid QD ensembles is still required to be well understood in order to fully utilize MEs in real-life applications of solid-state devices.

In this work, we present the spectral-temporal behavior of biexcitons (BXs) in the presence of photocharging in near-unity quantum yield (QY) $\mathrm{CdSe} / \mathrm{CdS}$ core/shell nanocrystals. By studying three QD samples with different core radii, we observe the spectral behavior modifications of the BXs as the result of strength tunability of $\mathrm{X}-\mathrm{X}$ interactions in the $\mathrm{CdSe} / \mathrm{CdS}$ QDs with size variation. Resolution of the recombination events of Xs, BXs, and trions in our ensemble measurements was achieved with time-correlated singlephoton counting (TCSPC) experiments under variable excitation intensities at different emission wavelengths. In order to extract $\mathrm{X}, \mathrm{BX}$, and trion decays, we developed an analysis approach for the time decays of the sample where we described the physical events happening in the QD ensemble with their corresponding time decay terms having specific lifetimes. This method, which uses the decay lifetime differences of different events to discriminate other events, provides us with the ability to distinguish the spectral behaviors of Xs, BXs, and trions, even when their spectral behaviors are almost the same, by tracing the corresponding decay terms throughout the spectrum. As a result, the spectral radiative recombination kinetics of $\mathrm{Xs}, \mathrm{BXs}$, and trions were obtained. Moreover, we verified our

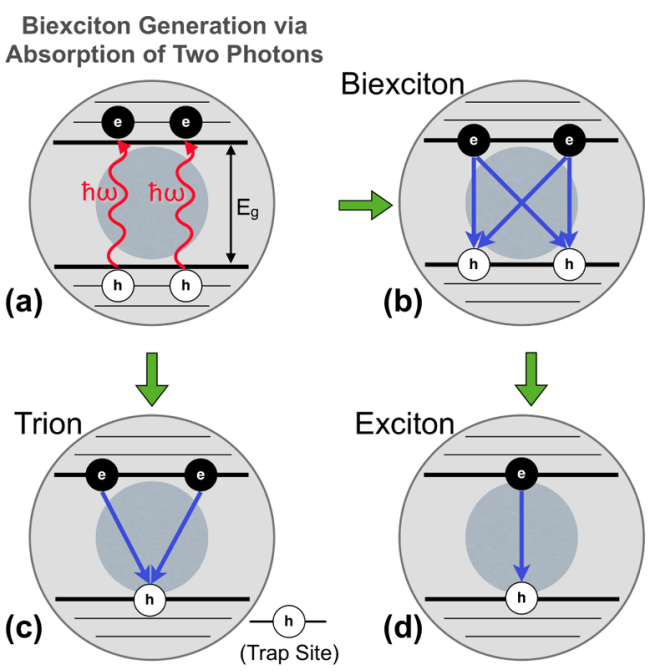

Figure 1. (a) Biexciton generation in a QD via absorption of two photons with energies $\hbar \omega$. (b) Biexciton formation immediately after the relaxation of excitons to the band edge. (The blue arrows indicate the possible recombination pathways.) (c) Trion composed of a hole and two electrons. The other hole is trapped at a trap site of the QD. (d) Exciton in a QD. This could be generated by direct absorption of a single photon or after the recombination of a BX. (The gray circles represent a core/shell QD. " $e$ " denotes an electron and " $h$ " denotes a hole. " $E_{\mathrm{g}}$ " stands for the band gap energy of the QD.).

results by cross-checking the excitation intensity dependences of these recombination events, evolutions of the photoluminescence spectra with time, independent steady-state photoluminescence behaviors, and exposure time dependences, all of which proved to be consistent with each other. In addition to the experimental results, the $\mathrm{X}$ and $\mathrm{BX}$ energies were estimated by standard second-order perturbation theory. The simulation results obtained are found to be in good agreement with the experimental measurements.

\section{RESULTS AND DISCUSSION}

MEs can be generated in a QD either by absorption of a highly energetic single photon followed by carrier multiplication (CM) process or by sequential absorption of multiple photons in a single pulse with lower photon energies. ${ }^{36}$ As illustrated in Figure $1 \mathrm{a}$, we generated MEs using the latter method. Since the behavior of MEs generated by either method has been shown to be the same, ${ }^{28}$ the results of this study are valid also for the MEs generated by $\mathrm{CM}$.

The assemblies of CdSe/CdS QDs used in the experiments feature $88 \%$ photoluminescence quantum yield, which results from a very high degree of surface passivation and small density of defect sites in the QDs. These QDs were then embedded in poly(methyl methacrylate) (PMMA) at very low concentration in solid film samples. The very low concentration of QDs in PMMA allowed us to obtain very homogeneous QD distribution in the solid medium on the quartz substrate. Moreover, the Förster-type nonradiative energy 
transfer (NRET) among the QDs was suppressed because of the increased average interparticle distance (>50 nm) and high dielectric surrounding medium. In addition to the suppression of NRET, QD's having nearunity QY combined with low-temperature characterization ensure a high degree of suppression of other nonradiative and nonideal transitions caused by the interfacial and surface defects. Therefore, the resulting TCSPC decay curves were expected to consist mostly of radiative recombination terms.

This material combination of CdSe used as the core and $\mathrm{CdS}$ used as the shell has previously been shown to have a very good radiative MER behavior because of the suppression of AR in the case of a "giant" QD structure, where the core is surrounded by at least 8-10 monolayers of $\mathrm{CdS} .^{2,5,7}$ Nonradiative AR is substantially suppressed (but not necessarily completely) in the QDs studied in this work; the resulting MEs in a significant portion of QDs in the ensemble have performed radiative recombination similar to the MEs in the giant QDs reported with the same core/shell materials. The suppression of the AR process to some extent may be explained by the quasi type II nature of these studied QDs.

The experimental time-resolved fluorescence (TRF) results were analyzed by least $\chi^{2}$ fittings with multiexponential fitting

$$
A_{1} e^{-t / \tau_{1}}+A_{2} e^{-t / \tau_{2}}+A_{3} e^{-t / \tau_{3}}+A_{4} e^{-t / \tau_{4}}+\text { noise }
$$

without enforcing any specific lifetime in any of the analyses. The numerical fitting therefore identified the best matching sets of $\left(A_{i}, \tau_{i}\right)$. The fitting lifetime components of interest here are at least orders of magnitude longer than the excitation pulse width and the instrument response function. Therefore, the results can be considered as the response of the system to an impulse excitation.

The method of extraction of $\mathrm{X}, \mathrm{BX}$, and trion recombination events from the total time decay curves of the ensemble is based on the lifetime differences of these physical events taking place in the ensemble. In other words, the dynamic signatures of the events are used to distinguish one from the other when exploring the spectral distributions and excitation intensity dependences of these events. Therefore, each one of the exponential decay terms in the fitting eq 1 can be attributed to a specific recombination event happening inside the QD ensemble. The resulting lifetimes together with their associated coefficients may then be examined to obtain the desired spectral dynamic behavior of the corresponding event. To find the total number of occurrences of a radiative recombination event in the ensemble, the total number of photons emitted from the sample should also be known. Integration of each decay term with respect to time gives the total number of photons emitted as a result of the corresponding physical event having that characteristic lifetime. Therefore, once the fitting coefficients and lifetimes are identified, the spectral distributions and excitation intensity dependences of the events happening in the excitation volume of the sample can conveniently be extracted and studied. Since we do not expect the emissions due to different events to have different angular emission dependences, the integration in eq 2 can be considered as a sampling of the number of occurrences of the event represented by the decay term $A_{i} e^{-t / \tau_{i}}(i=1-3)$, which is simply equivalent to the product of the lifetime describing the specific event with its weighting coefficient. Mathematically speaking, it is then necessary to keep track of $A_{i} \tau_{i}$ as a function of emission wavelength, for each event $i$. Therefore, it is physically more meaningful to study and compare $A_{i} \tau_{i}(\lambda)$ spectra of different events, rather than $A_{i}(\lambda)$ for a specific $\tau_{i}$.

$$
\int A_{i} e^{-t / \tau_{i}} \mathrm{~d} t=A_{i} \tau_{i}
$$

This kind of lifetime extraction methodology using TCSPC measurements has enabled us to resolve the spectral dynamic behaviors of different recombination events that would otherwise be unresolvable in ensemble systems with other methods that do not exploit the lifetime differences between the events. For example, in our case, we extracted the spectral dynamic behaviors of three different events taking place in the QD ensemble even though they are not distinguishable in the steady-state PL measurements. Thus, this approach allows us to overcome the problems of inhomogeneous broadening of QD emissions when the spectral peak positions of the two events are very close to each other. With this method, the lifetimes of each event throughout the whole emission spectrum can be analyzed. Moreover, the spectral-, temporal-, and power-dependent behaviors of different events can further be obtained even when they are spectrally unresolvable in the ensemble system.

Figure 2a shows the TRF decay curves taken at the photoluminescence peak of the QD solid sample for low- and high-intensity excitation cases. The emergence of the third and fourth exponential components for the high-intensity excitation case can be seen here. The corresponding fitting coefficients and lifetimes are given in Table 1. After collecting the fluorescence decay curves of the QDs at all emission photon energies for the specific excitation intensities, we obtain the spectral distributions of the coefficients of each exponential term in the overall decay eq 1.

The normalized fitting coefficients of each term in the overall decay for the medium intensity excitation case are shown in Figure 3a, which is useful to observe the spectral shifts between the events. The coefficients are provided here in the normalized form because the absolute amplitudes of the coefficients themselves 

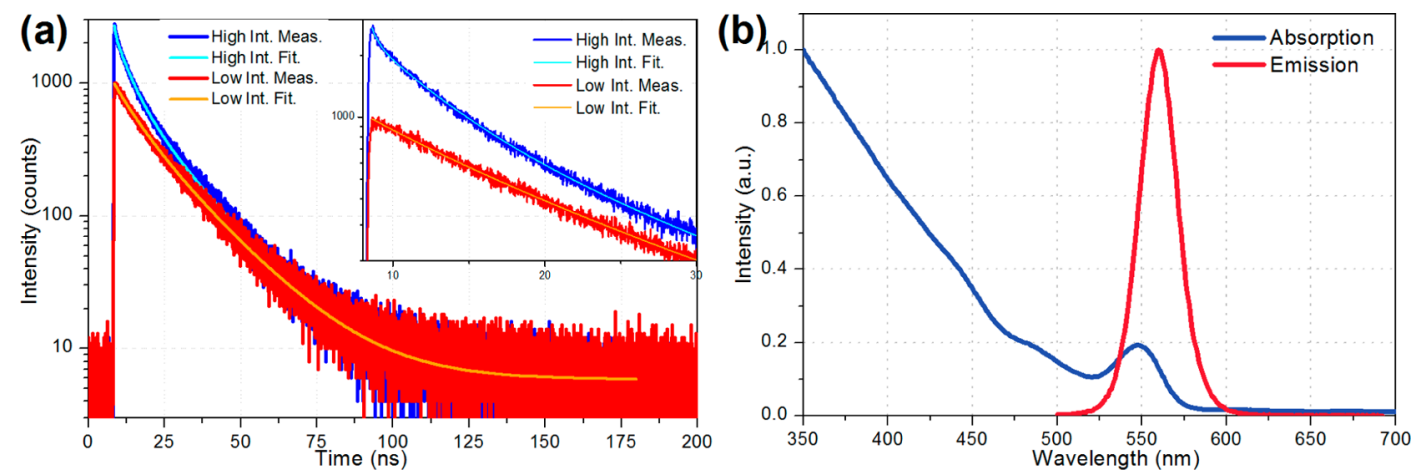

Figure 2. (a) TRF decays and fittings of the QD solid sample under low-intensity $\left(9.8 \times 10^{12}\right.$ photons $/ \mathrm{cm}^{2}$ per pulse and average number of absorbed photons per QD: $\langle N\rangle=J_{\mathrm{p}} \sigma_{\mathrm{abs}}=0.03$, where $J_{\mathrm{p}}$ is the per pump photon fluence and $\sigma_{\mathrm{abs}}$ is the QD absorption cross-section) and high-intensity $\left(5.1 \times 10^{14}\right.$ photons $/ \mathrm{cm}^{2}$ per pulse, $\left.\langle N\rangle=1.43\right)$ excitations collected for the same time duration given in red and blue curves, respectively. The inset gives the first $30 \mathrm{~ns}$ segments of these time decays. (b) Absorption and photoluminescence emission spectra of the in-solution QD sample.

TABLE 1. Fitting Coefficients and Lifetimes of High- and Low-Intensity Excitation Cases

\begin{tabular}{|c|c|c|c|c|c|c|c|c|}
\hline & $A_{1}$ & $\tau_{1}$ (ns) & $A_{2}$ & $\tau_{2}$ (ns) & $A_{3}$ & $\tau_{3}$ (ns) & $A_{4}$ & $\tau_{4}(\mathrm{~ns})$ \\
\hline low intens & $530 \pm 13$ & 18.48 & $372 \pm 26$ & 8.31 & $73 \pm 36$ & 4.60 & & \\
\hline high intens & $539 \pm 15$ & 18.99 & $559 \pm 28$ & 10.73 & $1218 \pm 56$ & 4.44 & $404 \pm 214$ & 0.41 \\
\hline
\end{tabular}
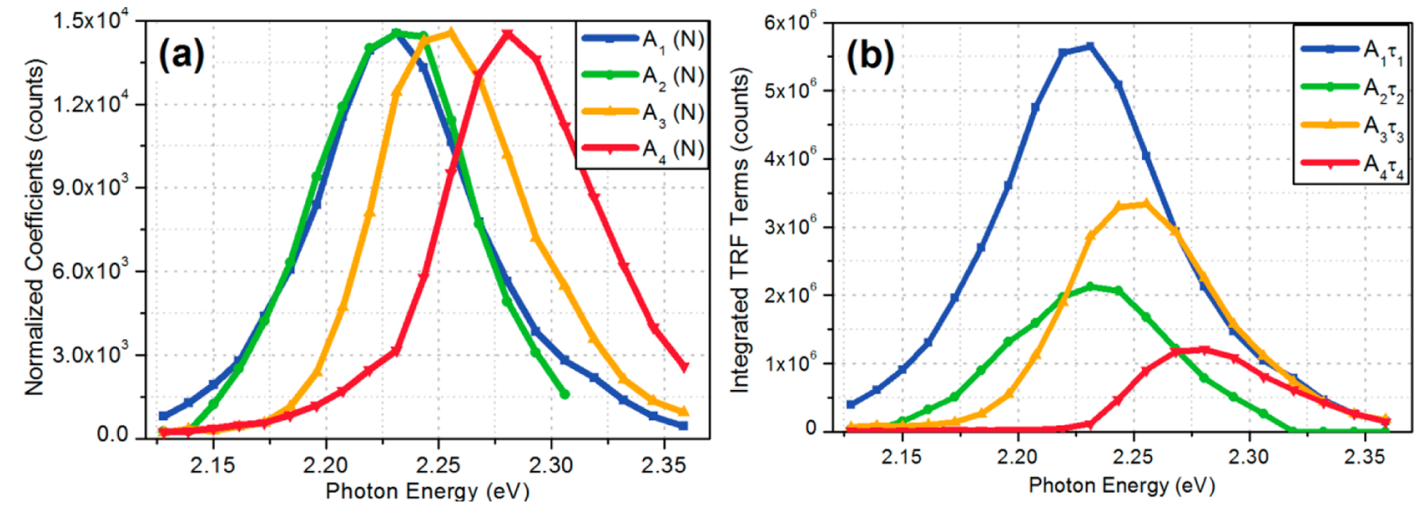

Figure 3. (a) Spectral distributions of normalized TRF fitting coefficients, $A_{i}(N)$, for events $i=1-4$. (b) Spectral distributions of TRF integrated fitting terms, $A_{i} \tau_{i}$ indicating the relative number of events taking place at a photon energy, for events $i=1-4$.

might be misleading, due to the fact that one needs to take the lifetimes into account to properly calculate the number of photons emitted as a result of that event (see the Supporting Information, Figure S1). Therefore, the coefficients themselves cannot be representative of the total number of times an event occurred, for which the radiative decay rates of the species should also be taken into account, as Padilha et al. have very recently considered in their transient cathodoluminescence results on the investigations of nonradiative decays of excitonic species in CdSe/ZnS QDs. ${ }^{37}$ A more accurate way of extracting the spectral distributions of the events is to utilize the integral in eq 2 (integrated TRF terms, $i=1-4$ in this case) instead of just the coefficients alone at all emission photon energies, as explained above. Therefore, the integrated TRF curves depicted in Figure $3 \mathrm{~b}$ are physically the most meaningful and important. These results presented in Figure $3 \mathrm{~b}$ look like steady-state photoluminescence (SS-PL) measurement results, but they cannot be obtained with a steady-state method because of the overlapping spectral distributions and hence spectral mixing of the corresponding events.

We also obtained the excitation intensity dependences of these events by collecting the spectral distributions of the integrated TRF terms at different excitation intensities. Figure 4a shows the dependences of the spectrally integrated total photon emissions of the corresponding events on the excitation intensity. As can be seen in Figure 4a, the total number of occurrences of the event that is represented by $A_{3}$ and $\tau_{3}$ exhibits nearly a quadratic excitation intensity dependence, while the event represented by $A_{1}$ and $\tau_{1}$ has a linear dependence. These characteristic behaviors are exactly what would be expected from BX and $X$ recombination events, respectively. The linear 

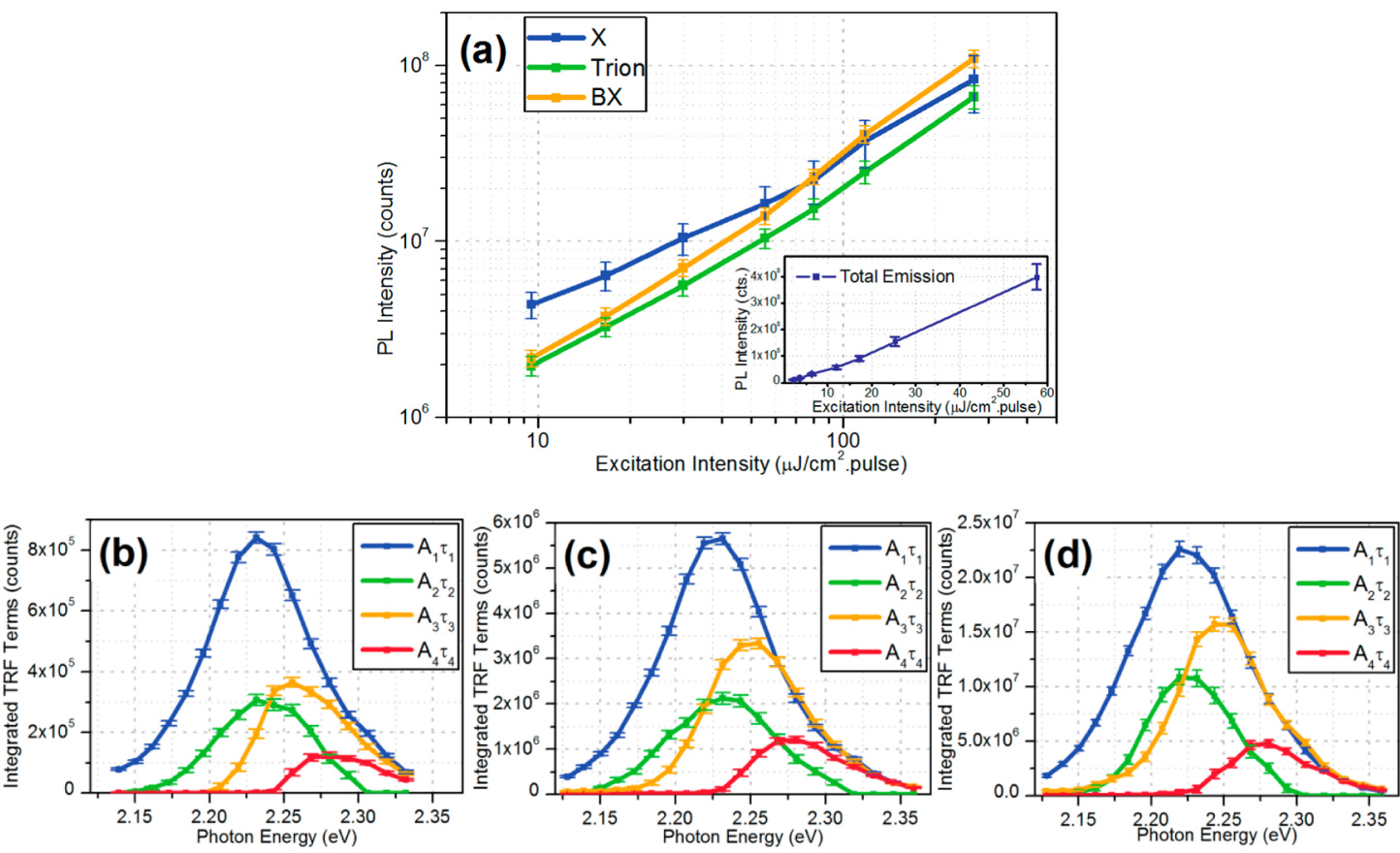

Figure 4. (a) Dependences of the total numbers of single exciton (X), trion, and biexciton (BX) recombination events on the excitation intensity. The inset gives the excitation intensity dependence of the total emission from the sample integrated spectrally and in time. (b-d) Spectral distributions of the integrated TRF terms for the lowest $\left(9.5 \mu \mathrm{J} /\left(\mathrm{cm}^{2}\right.\right.$ pulse), $\left.\langle N\rangle=0.05\right)$, medium $\left(80.2 \mu \mathrm{J} /\left(\mathrm{cm}^{2}\right.\right.$ pulse), $\left.\langle N\rangle=0.43\right)$, and highest $\left(268.1 \mu \mathrm{J} /\left(\mathrm{cm}^{2}\right.\right.$ pulse), $\left.\langle N\rangle=1.43\right)$ excitation intensity cases, respectively (see the Supporting Information, Figure S2).

dependence of $X$ recombination on the excitation intensity is straightforward, which basically comes from a single photon absorption generating a single $\mathrm{X}$ to recombine. The nearly quadratic dependence of $A_{3} \tau_{3}$ is, on the other hand, due to the cascaded absorptions of two photons within a single pulse by a single QD, which result in the formation of BXs. Therefore, the likelihood of forming a BX quadratically increases with increasing excitation intensity. In fact, this quadratic behavior is considered to be a lucid evidence of the biexcitonic origin of this decay component. ${ }^{2,27,38}$ (Here note that there is another component indicated as trions in Figure 4, which will be examined at a later point in the paper. Here it is worth noting that this component is growing with the excitation intensity faster than X's and slower than BX's.)

The spectra of each of the integrated TRF terms at the intensity levels used to construct Figure $4 a$ are provided in Figure $4 \mathrm{~b}-$ in the lowest, medium, and highest excitation intensity cases, respectively (see the Supporting Information). The three plots are scaled without changing the photon count numbers so that the $A_{1} \tau_{1}$ peaks are at the same level in order to guide the eye to the dominances of the integrated TRF terms. As can be seen from Figure $4 b-d$, as the excitation intensity increases, the $\mathrm{BX}$ recombination becomes more dominant, which is consistent with the trend given in Figure 4a. The increased dominance of the coefficient $A_{3}$ (see Table 1) of the TRF decay curve in Figure $2 a$ with the increased intensity is a result of the same fact that BXs become more dominant with the increased excitation intensity. It should also be noted here that, as shown in the inset of Figure 4a, the total emission from the sample has a linear excitation intensity dependence, suggesting that the features generated at higher intensities are also primarily radiative, pointing toward a significant suppression of Auger recombination.

The results of TRF measurements throughout the entire spectrum can also be analyzed by looking at the temporal cuts of the decay results at all wavelengths, as previously reported in the literature. ${ }^{3,39}$ Although the previous approach gives less information about the multiexcitonic behavior of the sample being tested in comparison to the method used in this paper, the former is easier to conduct and understand than the latter. Temporal evolution of the emission spectra of the sample after the laser pulse excitation is given in the inset of Figure 5. As can be seen in the figure, the PL spectrum shifts toward longer wavelength as time passes after the excitation pulse hits the sample. This shift results from the $B X$ emission, which takes place in the blue tail of the $X$ peak and has a shorter lifetime in comparison to the $X$ emission. Therefore, the emissions from BX states of the QDs diminish before the emissions from $X$ states do and the total emission spectrum shifts to the $X$ emission side over time. For a quantitative analysis, the shift of the PL peak position in time is depicted in Figure 5. If the recombination of the BXs in all of the QDs were totally nonradiative via an AR 


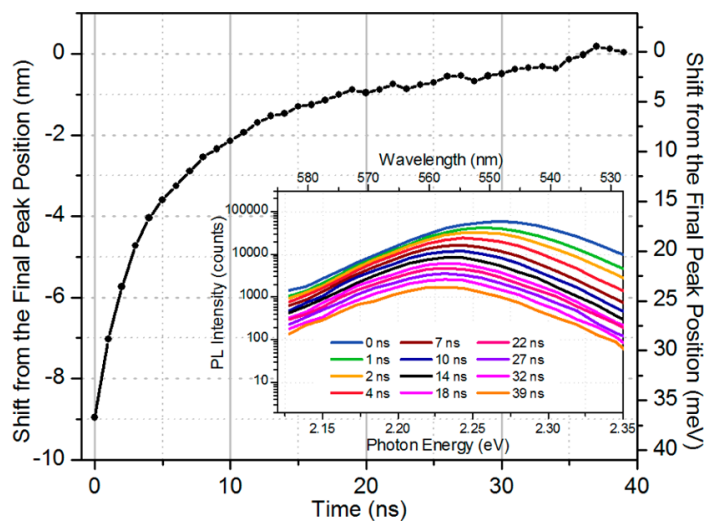

Figure 5. Time dependence of the PL peak position shift with respect to the final position under the excitation fluence of $2.6 \times 10^{14}$ photons $/\left(\mathrm{cm}^{2}\right.$ pulse $)(\langle N\rangle=0.74)$. The inset gives the evolution of the PL spectrum with time.

process, the time needed for the PL peak to come to the final PL peak position, where the $\mathrm{X}$ emission takes place, would take 1-2 ns because of the very short subnanosecond lifetime of the AR process. However, it is shown in Figure 5 that it takes more than 20 ns for the $\mathrm{PL}$ peak to arrive at the eventual position where emissions are only from the $X$ states of the QDs. These results support the claims that BXs emit at higher photon energies in these QDs and possess a lifetime around 4-5 ns, as obtained with the TRF decay fitting results. The long lifetimes of $B X s$ in comparison to typical AR lifetimes of BXs support that AR is suppressed in a substantial portion of the QDs in the ensemble. This is consistent with the conclusion drawn from the linear intensity dependence of total emission from the sample (inset of Figure 4a).

In addition to Figure 5 , it is observed from Figure $4 \mathrm{~b}-\mathrm{d}$ that the $\mathrm{BX}$ transition takes place at higher photon energies in comparison to the $X$ transition. This positive interaction energy is evidence of the repulsive $X-X$ Coulomb interaction between the two excitons in these QDs. The repulsive nature of this interaction can be explained by the quasi type II carrier distributions inside these QDs. In CdSe/CdS QDs, since the conduction band offset for the electrons at the interface is about $0.3 \mathrm{eV}$, which is too small to totally confine the electrons inside the core, the electrons are delocalized over the entire nanocrystal volume while the holes are mainly confined in the core, causing a local disturbance of the charge neutrality in the QDs (see the Supporting Information for calculated electron and hole wave functions, given in Figure S4). ${ }^{40}$ Therefore, the $\mathrm{X}-\mathrm{X}$ Coulomb interaction for quasi type II QDs is expected to be slightly repulsive, which is also the case here and is in agreement with a previous report. ${ }^{23}$ The amount of the interaction energy, the spectral shift of BXs with respect to Xs, observed here is $\Delta_{\mathrm{XX}}=\hbar \omega^{\mathrm{XX}}$ $\hbar \omega^{X} \cong 30$ meV. To support our experimental results, we estimate the energy shift between the Xs and BXs by standard second-order perturbation theory. In such a case, the eigenenergies of the $\mathrm{X}$ and $\mathrm{BX}$ are

$$
E=E_{0}+\lambda\left\langle 0\left|H^{\prime}\right| 0\right\rangle+\lambda^{2} \sum_{i} \frac{\left|\left\langle 0\left|H^{\prime}\right| i\right\rangle\right|^{2}}{E_{0}-E_{i}}
$$

where $E_{0}$ is the unperturbed eigenenergy and $|0\rangle$ is the unperturbed eigenvector of the $e-h$ ground state based on a kinetic energy, $E_{i}$ is the unperturbed eigenenergy and $|i\rangle$ is the unperturbed eigenvector of all the other states, and $H^{\prime}$ is the many-body perturbation Hamiltonian given by

$$
H^{\prime} \propto \frac{1}{2} \sum_{\alpha, \beta} q_{\alpha} q_{\beta} \frac{(\varepsilon-1)}{\varepsilon_{\mathrm{QD}} R_{\mathrm{QD}}} \sum_{i=1}^{\infty} \frac{1}{1+\varepsilon\left(\frac{i}{i+1}\right)}\left(\frac{r_{\alpha} r_{\beta}}{R_{\mathrm{QD}}^{2}}\right)^{i} P_{1}\left(\frac{r_{\alpha} \cdot r_{\beta}}{r_{\alpha} r_{\beta}}\right)
$$

where $\alpha$ and $\beta$ label two particles with charge $q, R_{\mathrm{QD}}$ is the core-shell radius, $r_{\alpha}$ and $r_{\beta}$ are the positions of the two charge particles, $P_{\mathrm{I}}$ is the Legendre polynomial function, and $\varepsilon=\varepsilon_{\mathrm{QD}} / \varepsilon_{\mathrm{M}}$ is the ratio between the $\mathrm{QD}$ dielectric constant $\varepsilon_{\mathrm{QD}}$ and the surrounding medium dielectric constant $\varepsilon_{\mathrm{M}}$. Note that we calculate the core-shell wave functions and use them to predict the energy shift. The computational result for the $\mathrm{X}-\mathrm{BX}$ energy shift (Coulomb interaction energy) is found $\Delta_{\mathrm{XX}}=E_{\mathrm{XX}}-2 E_{\mathrm{X}} \cong 33 \mathrm{meV}\left(E_{\mathrm{XX}}\right.$ and $E_{\mathrm{X}}$ are the calculated $B X$ and $X$ energies, respectively, and are related via $\hbar \omega^{\mathrm{XX}}=E_{\mathrm{XX}}-E_{\mathrm{X}}$ ), which is in very good agreement with the experimental value of $30 \mathrm{meV}$ (see the Supporting Information).

In addition to the quadratic intensity dependence and coherent spectral behavior of the decay term $A_{3} \tau_{3}$, the lifetime of this component is also a strong evidence for the biexcitonic origin of this component. Throughout the entire spectrum, for all excitation intensity levels, the lifetime of this component, $\tau_{3}$, is found to be very close to the one-fourth of the single exciton recombination lifetime, $\tau_{1}$ (see the Supporting Information). This ratio of $\mathrm{BX}$ radiative recombination rate to the $X$ recombination rate is expected to be 4 according to the quadratic scaling of the recombination rates with the exciton multiplicity, which was previously proposed and supported through "free carrier model" by McGuire et al. and also by some other reports. ${ }^{20,41}$ The reason behind the quadratic scaling of recombination rates is that there are $N^{2}$ possible recombination pathways for a QD with $N$ excitons in it, as illustrated in Figure 1. Therefore, the "free carrier model" can be said to hold true for this core/shell material combination both for the giant QDs reported previously ${ }^{2}$ and for these QDs reported here. The range of BX recombination lifetime and the clear shift of the photoluminescence spectra with time suggest that the recombination events of BXs through the TRF fitting term $A_{3} \tau_{3}$ are radiative and take place in the $Q D$ s that exhibit AR suppression behavior. Although AR suppression behavior is clearly present in the ensemble, there may 

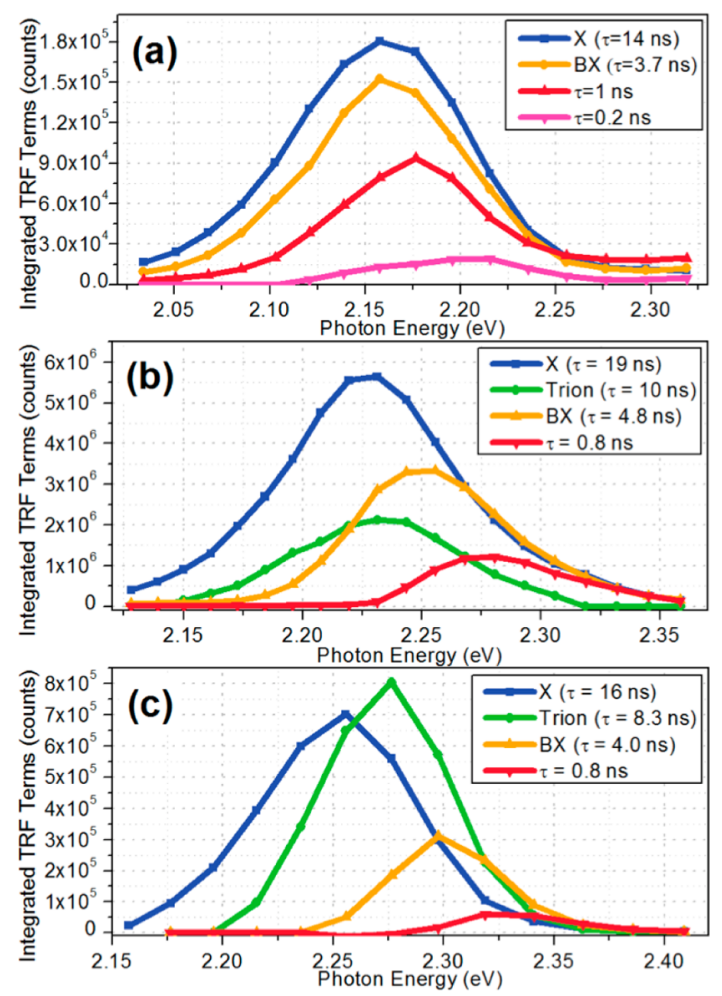

Figure 6. Spectral behavior of the integrated TRF terms of three different size QDs with core radii of $c a$. (a) $1.80 \mathrm{~nm}$, (b) $1.22 \mathrm{~nm}$, and (c) $1.10 \mathrm{~nm}$. The shell thicknesses of the QDs are ca. $1.4 \mathrm{~nm}$. The excitation fluences for all the cases are $5.1 \times 10^{14}$ photons $/\left(\mathrm{cm}^{2}\right.$ pulse $)$.

be also a subensemble of QDs that does not exhibit AR suppression in our sample, as is the case for some previous reports in the literature. ${ }^{1,5,41}$ If there exists, the recombination dynamics of BXs generated in this subensemble of QDs without AR suppression, i.e., the nonradiative part of the $B X$ dynamics, is included in the $A_{4} \tau_{4}$ term. This term has a subnanosecond lifetime, as expected from $A R$, and will be discussed later in the paper.

In addition to the QDs whose spectral $X$, trion, and BX behaviors are provided in Figure 4, we measured and obtained integrated TRF decay terms under highintensity excitation for two more CdSe/CdS QDs, one with a smaller core size and one with a larger core size, with both the same shell thicknesses as the previous middle-sized QD sample. As can be seen in Figure 6a, the spectra for the QDs with the largest core, the BX peak is almost at the same spectral position as the $X$ peak. The $B X-X$ peak shift is 30 and $43 \mathrm{meV}$ for the middle-sized (Figure $6 \mathrm{~b}$ ) and smallest core (Figure 6c) QDs, respectively. The reason for the trend of the higher energy $\mathrm{BX}$ formation in comparison to the Xs for the smaller-core QDs is that the delocalization of the electrons over the shell region of the QDs increases with a decrease in the core radius, which results in a more type II like behavior. For the large core case provided in Figure 6a, the local charge neutrality in the QDs is not distorted enough to observe a repulsive
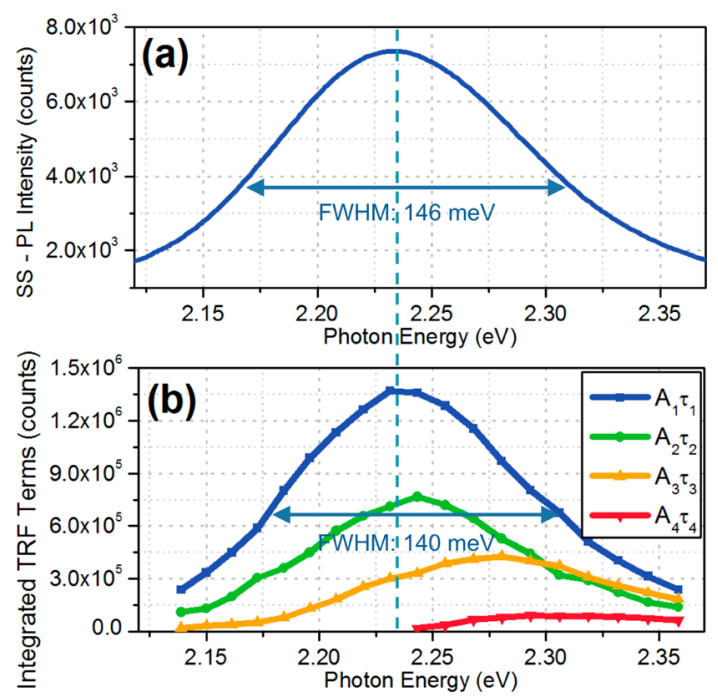

Figure 7. Consistency of the spectral behavior of the $A_{1} \tau_{1}$ term and steady-state PL spectrum: (a) steady-state PL spectrum of the sample; (b) spectral distributions of the integrated TRF decay terms under the excitation fluence of $5.1 \times 10^{14}$ photons $/\left(\mathrm{cm}^{2}\right.$ pulse) $\langle\langle N\rangle=1.43)$. The dashed vertical line emphasizes the peak positions of the spectra.

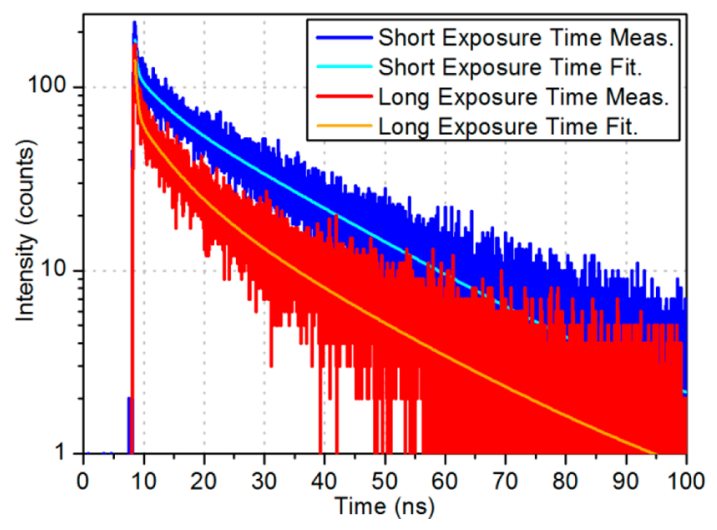

Figure 8. Experimental measurement and corresponding numerical fitting for the TRF decays of the QD sample at the PL emission peak under short and long exposure times to the laser excitation of $5.1 \times 10^{14}$ photons $/\left(\mathrm{cm}^{2}\right.$ pulse $)$ $(\langle N\rangle=1.43)$.

$\mathrm{X}-\mathrm{X}$ interaction and, hence, a blue-shifted $\mathrm{BX}$ with respect to the $X s$ is not observed.

In order to verify our claim that the $A_{1} \tau_{1}$ integrated TRF term is due to the emissions of the $X$ states of the QDs, we conducted TRF and SS-PL measurements at room temperature. In Figure 7, the spectral distributions of the integrated TRF terms under excitation intensity high enough to generate BXs and trions are compared to the SS-PL spectrum of the same sample taken under very low intensity continuous-wave excitation at $442 \mathrm{~nm}(3 \mathrm{eV})$. In the SS-PL measurement, we therefore expect only the emissions from the $X$ states of the QDs to contribute. Since we also attribute the TRF decay term represented by $A_{1} \tau_{1}$ to the emission of the $X$ states, one would expect a significant overlap of the $A_{1} \tau_{1}$ spectral distribution with the SS-PL spectrum. 
TABLE 2. Fitting Results of Short and Long Exposure Time Cases Plotted in Figure 8

$\begin{array}{lcccccccccc} & \boldsymbol{A}_{\mathbf{1}} & \tau_{\mathbf{1}} \text { (ns) } & \boldsymbol{A}_{\mathbf{2}} & \tau_{\mathbf{2}} \text { (ns) } & \boldsymbol{A}_{\mathbf{3}} & \tau_{\mathbf{3}} \text { (ns) } & \boldsymbol{A}_{\mathbf{4}} & \tau_{\mathbf{4}} \text { (ns) } & \text { total count } & \text { av lifetime (ns) } \\ \text { short exposure } & 82 \pm 3 & 23.2 & & & 36 \pm 12 & 4.81 & 63 \pm 54 & 0.323 & 1.52 \times 10^{5} & 11.59 \\ \text { long exposure } & 26 \pm 2 & 23.7 & 14 \pm 5 & 11.09 & 31 \pm 7 & 5.08 & 71 \pm 36 & 0.381 & 7.26 \times 10^{4} & 6.77\end{array}$

The results show almost full overlap, as expected. The consistency between totally independent TRF and SS-PL measurements in terms of $\mathrm{X}$ emission can be considered as a strong evidence of the $A_{1} \tau_{1}$ attribution to the $X$ decay.

In the previous parts where we discussed the BX and $X$ recombination events, the results for the attribution of $A_{1} \tau_{1}$ and $A_{3} \tau_{3}$ terms to $\mathrm{X}$ and $\mathrm{BX}$ recombination events were provided. Figure 8 presents the necessary information for us to make comments on the remaining two components of the overall TRF decay, $A_{2} \tau_{2}$ and $A_{4} \tau_{4}$.

Figure 8 shows two TRF decays of the QD solid sample under exactly the same excitation conditions for the same data collection duration only using different times of exposure to the laser beam. The blue curve is the case when the sample is exposed to the light for a few seconds, whereas the red curve is the decay curve of the sample after exposure to the laser beam for $15 \mathrm{~min}$. The corresponding fitting parameters are given in Table 2. For this experiment, we expect the radiative and nonradiative recombination events of charged QDs to become more dominant for the case of longer exposure time because of the accumulation of the charged QDs in the ensemble. Especially, the radiative and nonradiative decay terms of trions from the QDs with and without AR suppression, respectively, are expected to dominate. As can be seen from Figure 8 and Table 2, for the longer exposure time case, the total emission of the QDs decreases considerably because of the increased amount of nonradiative AR, which is enhanced due to the photocharging of the QDs. Since it is well established that the typical AR lifetimes for these kinds of QDs are on the order of hundreds of picoseconds, we expect to have a more pronounced coefficient of the decay term with a lifetime of 300-400 ps, which is $A_{4}$. The increase of the $A_{4}$ coefficient with exposure time, as shown in Table 2, means that at least a good portion of the 300-400 ps lifetime component is due to the $A R$ of trions. (Despite the fact that the experimental time resolution is not high enough to obtain exact lifetimes and coefficients of $A R$, we could still obtain conclusive results and make qualitative comments about the dominance of the corresponding decay component.) In addition to the nonradiative $\mathrm{AR}$ of trions, we observe an increase in the coefficient $A_{2}$, which we believe is the coefficient related to the radiative recombination of trions in the QDs with suppressed AR behavior. The trions with radiative recombination behavior are believed to be negatively charged, because the AR probability for positively charged trions is higher due to the high degree of spatial confinement for holes in this material system. ${ }^{42}$ The lifetime value, $\tau_{2}$, is ca. half of the lifetime of the $\mathrm{X}$ decay, $\tau_{1}$. This is another supporting result for the claim that $A_{2} \tau_{2}$ corresponds to the radiative decay of trions, consistent with the free carrier model ${ }^{20}$ and other reports in the literature. ${ }^{43}$ The observation of radiative recombinations of trions from the ensemble is considered to be a good sign of substantially suppressed AR. ${ }^{26}$ Also, in Figure 4, it is observed that the total number of occurrences of trion recombinations represented by the spectral integration of $A_{2} \tau_{2}$ exhibits a subquadratic and superlinear dependency on the excitation intensity, which is yet more supporting evidence for the observation of trions.

The last decay term that was observed in the TRF measurements is the $A_{4} \tau_{4}$ decay term, which we attribute to the nonradiative recombinations of trions, $\mathrm{BX}$, charged BXs, and species of higher multiplicity that are generated in the QDs without AR suppression behavior, whereas the first three decay terms were purely radiative. In our study, we do not observe a significant radiative recombination from the charged $B X$ states in the QD ensemble. This could be due to the higher dominancy of $A R$, in accordance with the very strong positive dependence of AR rate on the charge carrier number in the QDs, which makes the radiative recombination from charged BX states less likely. When the temperature was decreased to cryogenic temperatures, we observed that the $A_{4} \tau_{4}$ decay term became more pronounced at all emission photon energies. This shows that, in addition to the nonradiative AR of BXs and trions, the $A_{4} \tau_{4}$ decay term has some contribution from the emission of the singlet states before the system comes to a thermal equilibrium between singlet and triplet states. ${ }^{44,45}$ Further details of this very fast component were not investigated in this study and are out of the scope of this work. However, with higher time resolution characterization, the spectral and temporal behavior of these very fast events could also in principle be resolved with the systematic method employed here. The important point regarding this very fast weak component for our work here is that this component does not affect the results we obtained and conclusions we drew about the other terms of the overall decay.

\section{CONCLUSIONS}

In conclusion, we successfully resolved and identified the radiative $\mathrm{BX}$ recombination events in the 
presence of photocharging in the solid ensembles of CdSe/CdS QDs with near-unity QY. We distinguished the radiative $\mathrm{X}, \mathrm{BX}$, and trion recombination events by systematically analyzing the multiexponential decay components of the overall TRF decay throughout the entire emission spectrum. Our experiments on three different QD samples with different core sizes revealed the dependence of spectral BX recombination behavior on the carrier localizations in the QDs, which can be conveniently tuned to engineer the $\mathrm{X}-\mathrm{X}$ interaction energy. We introduced the integrated TRF terms $A_{i} \tau_{i}$ to reveal the spectrally resolved kinetics of multiexciton recombination, which is otherwise spectrally unresolved in the ensemble of QD solids. The spectral overlap of different events in MER normally makes the ensemble study of these events impossible, and single QD measurements would be needed in order to resolve and see the distinct spectral behaviors of different events. However, the analysis method devised here has enabled us to eliminate "the ensembling effect", because we relied on the characteristic decay lifetimes of the events to distinguish them from one another. Therefore, we could see the individual spectral behaviors of the events, even though they are spectrally mixed.

On the basis of this analysis methodology, for the TRF term $A_{3} \tau_{3}$, we obtained the quadratic excitation intensity dependence, TRF decay lifetime values quarter of the longest lifetime, and the temporal evolution of the PL spectra with a characteristic blue shift (due to the repulsive $\mathrm{X}-\mathrm{X}$ Coulomb interaction), which was also theoretically computed by the standard secondorder perturbation theory. These findings consistently strongly support the hypothesis that this $A_{3} \tau_{3}$ decay component results from the radiative recombination of BXs in the QDs with suppressed AR behavior. After the BX discussion, the independent SS-PL spectrum under very low intensity continuous-wave excitation was compared to the spectral distribution of the $A_{1} \tau_{1}$ component, showing that this component is indeed the $\mathrm{X}$ radiative decay component, in addition to the supporting evidence of the linear excitation intensity dependence. Finally, the exposure time dependences of $A_{2} \tau_{2}$ and $A_{4} \tau_{4}$ terms are used as experimental evidence for the attributions of these components to the radiative and nonradiative recombinations of trions, respectively, along with the supporting argument of subquadratic and superlinear excitation intensity dependence of $A_{2} \tau_{2}$, very short $\tau_{4}$ (hundreds of picoseconds), and $\tau_{2}$ being half of the longest lifetime. The observations of these radiative $\mathrm{BX}$ and trion recombinations indicate the suppression of Auger recombination in at least a good portion of these QDs in our ensemble samples.

We believe that these findings and discussions fill an important gap in understanding the spectral dynamics of MER in these QD solids and their behavior under the photocharging effects. In addition to the results and conclusions about BXs, trions, and Xs in QDs in this work, we feel that the systematic analysis approach devised in this study could be an important enabling tool that could help us study not only the radiative recombination events in any kind of ensemble samples consisting of one type of emitters but also their nonradiative recombination events. The only requirement for multiple events to be investigated in this method is that each event should have a unique decay lifetime, which is almost always ensured if the ensemble is of only one emitter type (and not a mixture of emitters).

The fundamental understanding of multiexciton recombinations together with the means to identify $B X s$, trions, and Xs paves the way to engineer MER processes to most effectively exploit the MEG concept in nanocrystal photovoltaics and lasing.

\section{METHODS}

Synthesis of Quantum Dots. The synthesis of CdSe/CdS QDs having a core radius of $c a .1 .22 \mathrm{~nm}$ and shell thickness of $c a$. $1.4 \mathrm{~nm}$ was carried out through a modified selective ion layer adsorption and reaction (SILAR) technique, as Greytak et al. previously reported. ${ }^{6,46}$ In this synthesis, CdSe cores were formed. Then, the $\mathrm{CdS}$ shell layers were grown around the cores at $180^{\circ} \mathrm{C}$, which is not high enough for an alloying to occur. Therefore, formation of interfacial alloy layers of CdSeS is not expected in this synthesis. The size distribution of the QDs obtained with this synthesis method is narrow $(<10 \%$ deviation from the average QD size), as can be seen in the transmission electron microscopy image of the QDs given in Figure S6. The PL full-width at half-maximum value of $c a .25 \mathrm{~nm}$ also indicates the highly monodisperse size distribution of the QDs.

Preparation of Quantum Dot Film Samples. The near-unity quantum yield $\mathrm{CdSe} / \mathrm{CdS}$ core/shell QDs were dispersed at a medium concentration in hexane. Then, $2 \mu \mathrm{L}$ of the QD solution was mixed with $98 \mu \mathrm{L}$ of poly(methyl methacrylate) (PMMA) A7.5 with the help of a Vortex mixer (Velp Scientifica Inc.) for approximately $5 \mathrm{~min}$ until QDs were dispersed completely homogeneously inside the PMMA. A $50 \times$ dilution of the QD solution ensured the very low concentration of QD solids in order to avoid nonradiative energy transfer in the sample. A $25 \mu \mathrm{L}$ portion of this QD-PMMA mixture was spin-coated onto clean quartz substrates of $1 \mathrm{~cm} \times 1 \mathrm{~cm}$ in dimensions at a $1500 \mathrm{rpm}$ rotation speed for $3 \mathrm{~min}$.

Absorption Cross-Section Calculations. The absorption cross-section, $\sigma_{\text {abs }}$ of the core/shell QDs at $375 \mathrm{~nm}$ excitation wavelength is obtained by comparing the absorbance of an as-synthesized in-solution core/shell sample to that of the same concentration core sample before the shell coating. From the previously reported molar extinction coefficient of CdSe QDs, ${ }^{47}$ we obtained $\sigma_{\text {abs }}=2.83 \times 10^{-15} \mathrm{~cm}^{2}$.

Time-Resolved Fluorescence Measurements. We used a $3.3 \mathrm{eV}$ $(375 \mathrm{~nm})$ pulsed laser excitation with a pulse width of less than $50 \mathrm{ps}$ at a repetition rate of $5 \mathrm{MHz}$ which is focused to a spot size of $37 \mu \mathrm{m}^{2}$ on the sample for the TCSPC measurements (Picoquant Fluotime 200). Laser fluences were varied from $9.8 \times 10^{12}$ to $5.1 \times 10^{14}$ photons $/\left(\mathrm{cm}^{2}\right.$ pulse $)$. The collection part of the TRF setup consists of a monochromator with $8 \mathrm{~nm}$ wavelength resolution and $0.3 \mathrm{~nm}$ wavelength accuracy and a 
photomultiplier tube with controlling electronics, resulting in a time resolution of $c a$. 200 ps. To avoid phonon-mediated undesired processes as much as possible, we worked at temperatures below $20 \mathrm{~K}$ with the help of a closed-cycle liquid $\mathrm{He}$ cryostat (Cryo Industries).

The TRF decay curves were fitted with the multiexponential fitting formula using the least $\chi^{2}$ fitting algorithm. Since the best $\chi^{2}$ values were obtained for the four-exponential fittings, the decay curves were fitted with the four exponentials. We also checked the resulting spectral behaviors with three exponentials, which resulted in physically meaningless steep changes in the individual lifetime values for the varying emission wavelengths. The consistency of the lifetime values and ratios of each decay term to each other throughout the entire spectrum, as provided in Figure S5 (Supporting Information), also justifies that each decay term represents a physical event. For the fiveexponential fitting trials, the fifth decay coefficient turned out to be zero, justifying our four-exponential fittings.

Conflict of Interest: The authors declare no competing financial interest.

Acknowledgment. This work was supported in part by the Singapore National Research Foundation under the program numbers NRF-CRP-6-2010-02 and NRF-RF-2009-09 and in part by the ESF-EURYI. Also, H.V.D. gratefully acknowledges support from the TUBA-GEBIP. A.F.C. and Y.K. acknowledge support from the TUBITAK BIDEB.

Supporting Information Available: Figures, text, and tables giving absolute time-resolved fluorescence coefficients of the QD solid sample for the measurement presented in Figure 3, intensity dependences of exciton, trion, and biexciton numbers, spectral distributions of integrated TRF terms at intermediate power levels, derivations and calculations for the exciton and biexciton energies and wave functions, spectral distributions of the lifetimes of each decay term, size distribution of the QDs, and high-resolution transmission electron microscopy. This material is available free of charge via the Internet at http:// pubs.acs.org.

\section{REFERENCES AND NOTES}

1. Mahler, B.; Spinicelli, P.; Buil, S.; Quelin, X.; Hermier, J.-P.; Dubertret, B. Towards Non-blinking Colloidal Quantum Dots. Nat. Mater. 2008, 7, 659-664.

2. Htoon, H.; Malko, A. V; Bussian, D.; Vela, J.; Chen, Y.; Hollingsworth, J. A.; Klimov, V. I. Highly Emissive Multiexcitons in Steady-State Photoluminescence of Individual "Giant" CdSe/CdS Core/Shell Nanocrystals. Nano Lett. 2010, 10, 2401-2407.

3. Marceddu, M.; Saba, M.; Quochi, F.; Lai, A.; Huang, J.; Talapin, D. V; Mura, A.; Bongiovanni, G. Charged Excitons, Auger Recombination and Optical Gain in CdSe/CdS Nanocrystals. Nanotechnology 2012, 23, 015201.

4. García-Santamaría, F.; Brovelli, S.; Viswanatha, R.; Hollingsworth, J. A; Htoon, H.; Crooker, S. A.; Klimov, V. I. Breakdown of Volume Scaling in Auger Recombination in $\mathrm{CdSe} / \mathrm{CdS}$ Heteronanocrystals: The Role of the Core-shell Interface. Nano Lett. 2011, 11, 687-693.

5. Park, Y.-S.; Malko, A.; Vela, J.; Chen, Y.; Ghosh, Y.; GarcíaSantamaría, F.; Hollingsworth, J.; Klimov, V. I.; Htoon, H. Near-Unity Quantum Yields of Biexciton Emission from CdSe/CdS Nanocrystals Measured Using Single-Particle Spectroscopy. Phys. Rev. Lett. 2011, 106, 187401.

6. Greytak, A. B.; Allen, P. M.; Liu, W.; Zhao, J.; Young, E. R.; Popović, Z.; Walker, B. J.; Nocera, D. G.; Bawendi, M. G. Alternating Layer Addition Approach to CdSe/CdS Core/ shell Quantum Dots with Near-unity Quantum Yield and High On-time Fractions. Chem. Sci. 2012, 3, 2028-2034.

7. García-Santamaría, F.; Chen, Y.; Vela, J.; Schaller, R. D.; Hollingsworth, J. A.; Klimov, V. I. Suppressed Auger Recombination in "Giant" Nanocrystals Boosts Optical Gain Performance. Nano Lett. 2009, 9, 3482-3488.

8. Osovsky, R.; Cheskis, D.; Kloper, V.; Sashchiuk, A.; Kroner, M.; Lifshitz, E. Continuous-Wave Pumping of Multiexciton
Bands in the Photoluminescence Spectrum of a Single CdTe-CdSe Core-Shell Colloidal Quantum Dot. Phys. Rev. Lett. 2009, 102, 197401.

9. Gachet, D.; Avidan, A.; Pinkas, I.; Oron, D. An Upper Bound to Carrier Multiplication Efficiency in Type II Colloidal Quantum Dots. Nano Lett. 2010, 10, 164-170.

10. Oron, D.; Kazes, M.; Banin, U. Multiexcitons in type-II Colloidal Semiconductor Quantum Dots. Phys. Rev. B 2007, 75, 035330.

11. Semonin, O. E.; Luther, J. M.; Choi, S.; Chen, H.-Y.; Gao, J.; Nozik, A. J.; Beard, M. C. Peak External Photocurrent Quantum Efficiency Exceeding 100\% via MEG in a Quantum Dot Solar Cell. Science 2011, 334, 1530-1533.

12. Sambur, J. B.; Novet, T.; Parkinson, B. A. Multiple Exciton Collection in a Sensitized Photovoltaic System. Science 2010, 330, 63-66.

13. Klimov, V. I. Detailed-balance Power Conversion Limits of Nanocrystal-quantum-dot Solar Cells in the Presence of Carrier Multiplication. Appl. Phys. Lett. 2006, 89, 123118.

14. Kim, S. J.; Kim, W. J.; Cartwright, A. N.; Prasad, P. N. Carrier Multiplication in a PbSe Nanocrystal and P3HT/PCBM Tandem Cell. Appl. Phys. Lett. 2008, 92, 191107.

15. Klimov, V. I. Mechanisms for Photogeneration and Recombination of Multiexcitons in Semiconductor Nanocrystals: Implications for Lasing and Solar Energy Conversion. J. Phys. Chem. B 2006, 110, 16827-16844.

16. Hanna, M. C.; Nozik, A. J. Solar Conversion Efficiency of Photovoltaic and Photoelectrolysis Cells with Carrier Multiplication Absorbers. J. Appl. Phys. 2006, 100, 074510.

17. Nozik, A. J.; Beard, M. C.; Luther, J. M.; Law, M.; Ellingson, R. J.; Johnson, J. C. Semiconductor Quantum Dots and Quantum Dot Arrays and Applications of Multiple Exciton Generation to Third-generation Photovoltaic Solar Cells. Chem. Rev. 2010, 110, 6873-6890.

18. Schaller, R. D.; Klimov, V. I. High Efficiency Carrier Multiplication in PbSe Nanocrystals: Implications for Solar Energy Conversion. Phys. Rev. Lett. 2004, 92, 186601.

19. Beard, M. C. Multiple Exciton Generation in Semiconductor Quantum Dots. J. Phys. Chem. Lett. 2011, 2, 1282-1288.

20. McGuire, J. A; Joo, J.; Pietryga, J. M.; Schaller, R. D.; Klimov, V. I. New Aspects of Carrier Multiplication in Semiconductor Nanocrystals. Acc. Chem. Res. 2008, 41, 1810-1819.

21. Nozik, A. J. Multiple Exciton Generation in Semiconductor Quantum Dots. Chem. Phys. Lett. 2008, 457, 3-11.

22. Klimov, V. I.; Mikhailovsky, A. A.; Xu, S.; Malko, A.; Hollingsworth, J. A.; Leatherdale, C. A.; Eisler, H.-J.; Bawendi, M. G. Optical Gain and Stimulated Emission in Nanocrystal Quantum Dots. Science 2000, 290, 314-317.

23. Klimov, V. I.; Ivanov, S. A.; Nanda, J.; Achermann, M.; Bezel, I.; McGuire, J. A.; Piryatinski, A. Single-exciton Optical Gain in Semiconductor Nanocrystals. Nature 2007, 447, 441-446.

24. Dang, C.; Lee, J.; Breen, C.; Steckel, J. S.; Coe-Sullivan, S.; Nurmikko, A. Red, Green and Blue Lasing Enabled by Single-exciton Gain in Colloidal Quantum Dot Films. Nat. Nano. 2012, 7, 335-339.

25. Sukhovatkin, V.; Hinds, S.; Brzozowski, L.; Sargent, E. H. Colloidal Quantum-dot Photodetectors Exploiting Multiexciton Generation. Science 2009, 324, 1542.

26. Wang, X.; Ren, X.; Kahen, K.; Hahn, M. a; Rajeswaran, M.; Maccagnano-Zacher, S.; Silcox, J.; Cragg, G. E.; Efros, A. L.; Krauss, T. D. Non-blinking Semiconductor Nanocrystals. Nature 2009, 459, 686-689.

27. Louyer, Y.; Biadala, L.; Trebbia, J.-B.; Fernée, M. J.; Tamarat, P.; Lounis, B. Efficient Biexciton Emission in Elongated CdSe/ZnS Nanocrystals. Nano Lett. 2011, 11, 4370-4375.

28. Schaller, R. D.; Sykora, M.; Jeong, S.; Klimov, V. I. Highefficiency Carrier Multiplication and Ultrafast Charge Separation in Semiconductor Nanocrystals Studied via Timeresolved Photoluminescence. J. Phys. Chem. B 2006, 110, 25332-25338.

29. Pijpers, J. J. H.; Hendry, E.; Milder, M. T. W.; Fanciulli, R.; Savolainen, J.; Herek, J. L.; Vanmaekelbergh, D.; Ruhman, S.; Mocatta, D.; Oron, D.; et al. Carrier Multiplication and Its Reduction by Photodoping in Colloidal InAs Quantum Dots. J. Phys. Chem. C 2007, 111, 4146-4152. 
30. Pijpers, J. J. H.; Hendry, E.; Milder, M. T. W.; Vanmaekelbergh, D.; Ruhman, S.; Mocatta, D.; Oron, D.; Aharoni, A.; Banin, U.; Bonn, M. Additions and Corrections 2008. J. Phys. Chem. C 2008, 112, 4783-4784.

31. Nair, G.; Bawendi, M. G. Carrier Multiplication Yields of $\mathrm{CdSe}$ and CdTe Nanocrystals by Transient Photoluminescence Spectroscopy. Phys. Rev. B 2007, 76, 081304.

32. Nair, G.; Geyer, S.; Chang, L.-Y.; Bawendi, M. G. Carrier Multiplication Yields in PbS and PbSe Nanocrystals Measured by Transient Photoluminescence. Phys. Rev. B 2008, 78, 125325.

33. Mcguire, J. A.; Sykora, M.; Joo, J.; Pietryga, J. M.; Klimov, V. I. Apparent Versus True Carrier Multiplication Yields in Semiconductor Nanocrystals. Nano Lett. 2010, 10, 2049-2057.

34. Midgett, A. G.; Hillhouse, H. W.; Hughes, B. K.; Nozik, A. J.; Beard, M. C. Flowing Versus Static Conditions for Measuring Multiple Exciton Generation in PbSe Quantum Dots. J. Phys. Chem. C 2010, 114, 17486-17500.

35. Gesuele, F.; Sfeir, M. Y.; Murray, C. B.; Heinz, T. F.; Wong, C. W. Ultrafast Supercontinuum Spectroscopy of Carrier Multiplication and Biexcitonic Effects in Excited States of PbS Quantum Dots. Nano Lett. 2012, 12, 2658-2664.

36. Stewart, J. T.; Padilha, L. A.; Qazilbash, M. M.; Pietryga, J. M.; Midgett, A. G.; Luther, J. M.; Beard, M. C.; Nozik, A. J.; Klimov, V. I. Comparison of Carrier Multiplication Yields in PbS and PbSe Nanocrystals: The Role of Competing Energy-Loss Processes. Nano Lett. 2012, 12, 622-628.

37. Padilha, L. A.; Bae, W. K.; Klimov, V. I.; Pietryga, J. M.; Schaller, R. D. Response of Semiconductor Nanocrystals to Extremely Energetic Excitation. Nano Lett. 2013, 13, 925-932.

38. Sitt, A.; Sala, F. D.; Menagen, G.; Banin, U. Multiexciton Engineering in Seeded Core/shell Nanorods: Transfer from type-I to quasi-type-II Regimes. Nano Lett. 2009, 9, 34703476.

39. Gdor, I.; Sachs, H.; Roitblat, A.; Strasfeld, D. B.; Bawendi, M. G.; Ruhman, S. Exploring Exciton Relaxation and Multiexciton Generation in PbSe Nanocrystals Using Hyperspectral Near-IR Probing. ACS Nano 2012, 6, 3269-3277.

40. Talapin, D. V; Koeppe, R.; Go, S.; Kornowski, A.; Lupton, J. M.; Rogach, A. L.; Benson, O.; Feldmann, J.; Weller, H. Highly Emissive Colloidal CdSe/CdS Heterostructures of Mixed Dimensionality. Nano Lett. 2003, 3, 1677-1681.

41. Zhao, J.; Chen, O.; Strasfeld, D. B.; Bawendi, M. G. Biexciton Quantum Yield Heterogeneities in Single CdSe (CdS) Core (Shell) Nanocrystals and Its Correlation to Exciton Blinking. Nano Lett. 2012, 12, 4477-4483.

42. Galland, C.; Ghosh, Y.; Steinbrück, A.; Hollingsworth, J. A.; Htoon, H.; Klimov, V. I. Lifetime Blinking in Nonblinking Nanocrystal Quantum Dots. Nat. Commun. 2012, 3, 908.

43. Jha, P. P.; Guyot-Sionnest, P. Trion Decay in Colloidal Quantum Dots. ACS Nano 2009, 3, 1011-1015.

44. Wuister, S. F.; Koole, R.; Donega, C. D. M.; Meijerink, A. Temperature-Dependent Energy Transfer in Cadmium Telluride Quantum Dot Solids. J. Appl. Phys. 2005, 109, 5504.

45. Labeau, O.; Tamarat, P.; Lounis, B. Temperature Dependence of the Luminescence Lifetime of Single CdSe/ZnS Quantum Dots. Phys. Rev. Lett. 2003, 90, 257404.

46. Li, J. J.; Wang, Y. A.; Guo, W.; Keay, J. C.; Mishima, T. D.; Johnson, M. B.; Peng, X. Large-scale Synthesis of Nearly Monodisperse CdSe/CdS Core/shell Nanocrystals Using Air-stable Reagents via Successive Ion Layer Adsorption and Reaction. J. Am. Chem. Soc. 2003, 125, 12567-12575.

47. Leatherdale, C. A.; Mikulec, F. V.; Bawendi, M. G. On the Absorption Cross Section of CdSe Nanocrystal Quantum Dots. J. Phys. Chem. B 2002, 106, 7619-7622. 